\title{
Absolute instability in a traveling wave tube model
}

\author{
L. K. Ang and Y. Y. Lau \\ Department of Nuclear Engineering and Radiological Sciences, University of Michigan, Ann Arbor, \\ Michigan 48109-2104
}

(Received 15 June 1998; accepted 27 July 1998)

\begin{abstract}
A model is constructed to evaluate absolute instability which may lead to bandedge oscillations in a traveling wave tube. Under the assumptions (a) that all modes have forward group velocities, and (b) that the slow wave structure has a parabolic dispersion relation in the $\omega$ - $k$ plane, the threshold coupling constant (Pierce's parameter $C$ ) is calculated for the onset of absolute instability. The effect of distributed resistive loss in the circuit is included. The axial wave number and the characteristic frequency of the oscillation at the onset are given. (C) 1998 American Institute of Physics. [S1070-664X(98)00512-6]
\end{abstract}

\section{INTRODUCTION}

There is a rekindling of interest in traveling wave tubes (TWTs), due to the recent advances in microwave power module $^{1}$ and to the rapid growth in satellite communication, where TWTs are extensively used. In a TWT, the electron beam interacts with the forward wave of the slow wave circuit, resulting in spatial amplification of the signal. A serious threat to the stability of TWT is bandedge oscillation, ${ }^{2}$ characterized by a frequency in the vicinity of the $\pi$-mode of the slow wave circuit. A candidate for bandedge oscillation is the excitation of absolute instability, ${ }^{3}$ which may occur when the beam current exceeds some threshold value, even if all modes (beam and circuit) of interaction have forward group velocities. ${ }^{4}$ In this note, we present a calculation of this threshold current based on a simplified model of TWT.

In general, absolute instability can occur even if the TWT is perfectly matched at both ends. ${ }^{4}$ Thus, it is different from the regenerative oscillations caused by partial reflections of the amplified signal at the ends of the tube. ${ }^{2}$ It is also different from the backward wave oscillations that occur in the event that the beam mode intersects the circuit mode at a negative group velocity. ${ }^{5}$ While absolute instability has been predicted $^{4}$ and observed in great detail ${ }^{6}$ for the (fast wave) gyro-TWTs, we are not aware of similar calculations on the conventional (slow wave) TWTs. Toward the end of this paper, we briefly discuss the differences in absolute instabilities in fast wave and slow wave tubes.

The helix or ring-bar circuits in a conventional TWT $^{2}$ lead to a rather complicated dispersion relation for the circuit mode. To simplify the calculations of the threshold current for the onset of absolute instability, we assume that the circuit mode dispersion relation is a parabola in the $\omega-k$ plane (Fig. 1). This could be a reasonable assumption near the $\pi$-mode (bandedge), but is entirely inadequate to model over a much wider band that is typical of a TWT. ${ }^{1,7}$ Nevertheless, we choose the parameters in such a way that, for synchronous interaction (defined to be the case where the phase and group velocities are all equal in the beam mode and in the circuit mode), our simplified dispersion relation is identical to Pierce's standard form. ${ }^{2}$ In addition, the simplified disper- sion relation allows us to readily investigate the effects of velocity detune and of a distributed resistive loss in the circuit. When the resistive loss is absent, the threshold coupling constant (Pierce's parameter $C^{3}$ ), the frequency, and wave number at the onset of absolute instability are derived analytically.

\section{ANALYSIS}

In our model, electrons drift axially with a velocity $v$ inside a slow wave structure with pinch $L$ [Fig. 1(a)]. The beam mode is $\omega=k v$, with $\exp (j \omega t-j k z)$ dependence in the small signal regime [Fig. 1(b)]. We approximate the structure mode by a parabola $\omega=\omega_{\mathrm{BE}}-\alpha\left(k-k_{\mathrm{BE}}\right)^{2}$, where $k_{\mathrm{BE}}$ $=\pi / L$ is the wave number at bandedge, ${ }^{7} \omega_{\mathrm{BE}}$ is the bandedge frequency, and $\alpha$ determines the "shape" of the parabola. When $v=v_{0}$, the beam mode dispersion curve is tangent to the parabola at $\omega=\omega_{0}, k=k_{0}$ [Fig. 1(b)]. We call this case, $v=v_{0}$, the synchronous case. In terms of $k_{\mathrm{BE}}, \omega_{\mathrm{BE}}$ and $\alpha$, we find $k_{0}=\left(k_{\mathrm{BE}}^{2}-\omega_{\mathrm{BE}} / \alpha\right)^{1 / 2}, \omega_{0}=2 \alpha\left(k_{\mathrm{BE}}-k_{0}\right) /$ $k_{0}$, and $v_{0}=\omega_{0} / k_{0}$. Note that for $v>v_{\mathrm{BE}}$, where $v_{\mathrm{BE}}$ $=\omega_{\mathrm{BE}} / k_{\mathrm{BE}}$, all interacting modes have forward group velocities [Fig. 1(b)]. In this paper, we concentrate only on such cases.

By normalizing $\omega$ with respect to $\omega_{0}$ and $k$ to $k_{0}$, we model the TWT dispersion relation as ${ }^{8}$

$$
\begin{aligned}
& D(\bar{k}, \bar{\omega}) \equiv[\bar{k}(1+\delta)-\bar{\omega}]^{2}\left[P(\bar{k}-1)^{2}-(\bar{k}-\bar{\omega})+\Delta(1-j)\right] \\
& \quad=\varepsilon \\
& P=\frac{k_{0}}{2\left(k_{\mathrm{BE}}-k_{0}\right)} .
\end{aligned}
$$

Here, $\bar{\omega}=\omega / \omega_{0}, \bar{k}=k / k_{0}$, and $\varepsilon>0$ ( $\varepsilon \propto$ current $)$ is the dimensionless coupling constant. In Eq. (1), the first square bracket represents the beam mode and the second square bracket represents the structure mode, when these square brackets are set equal to zero individually. We have included the effects of beam detuning through $\delta=\left(v-v_{0}\right) / v_{0}$ (either positive or negative) in the beam mode, and of a distributed resistive loss through $\Delta(>0)$ in the structure mode. We take 


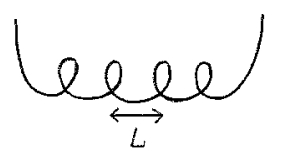

(a)

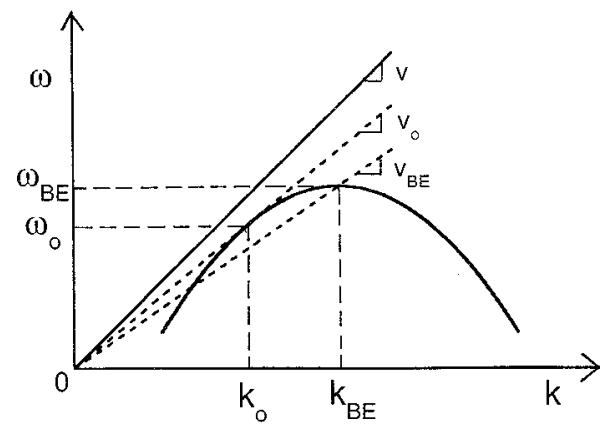

(b)

FIG. 1. (a) A helix of pitch $L$. (b) The beam mode $\omega=k v$ and the structure mode, approximated by a parabola. The $\pi$-mode wave number is $k_{\mathrm{BE}}$ $=\pi / L$.

the dimensionless parameters $\delta$ and $\Delta$ to be small, and 0 $<k_{0} / k_{\mathrm{BE}}<1$. In terms of $P$, we find $\bar{k}_{\mathrm{BE}} \equiv k_{\mathrm{BE}} / k_{0}=1$ $+1 / 2 P$ and $\bar{\omega}_{\mathrm{BE}} \equiv \omega_{\mathrm{BE}} / \omega_{0}=1+1 / 4 P$.

Note that the dimensionless coupling constant, $\varepsilon$, is essentially $C^{3}$, where $C$ is the Pierce parameter. ${ }^{2}$ To see this, take the synchronous, lossless limit $(\delta=0, \Delta=0)$. Upon approximating $\bar{k} \approx 1$, Eq. (1) then reduces to $(\bar{\omega}-\bar{k})^{3}=\varepsilon$ $=C^{3}$, which is Pierce's standard TWT dispersion relation for synchronous interaction. ${ }^{2}$ Furthermore, for synchronous interaction, the cold-tube dispersion relation yields $\bar{k} \approx \bar{\omega}$ $+\Delta(1-j)$ according to Eq. (1). Thus, $\Delta$ represents a distributed resistive loss on the circuit. Numerically, $\Delta$ $=Z_{L} v_{0} / 8.686 \omega_{0}$, where $Z_{L}$ is the cold-tube loss rate in $\mathrm{dB} / \mathrm{m}, v_{0}$ is the beam velocity in $\mathrm{m} / \mathrm{s}$, and $\omega_{0}$ is the frequency in radians/second.

We use the Briggs-Bers criterion ${ }^{3}$ to calculate the threshold value of $\varepsilon, \varepsilon_{s}$, for the onset of absolute instability for various combinations of $P, \delta, \Delta$. First, for a lossless structure $(\Delta=0)$, we use the techniques of Ref. 4 to show from Eq. (1) that both $\bar{\omega}=\bar{\omega}_{s}$ and $\bar{k}=\bar{k}_{s}$ are real at the onset of the absolute instability. We solve $\bar{\omega}_{s}$ and $\bar{k}_{s}$ by setting $\partial D / \partial \bar{k}=0, \partial^{2} D / \partial \bar{k}^{2}=0$ and choosing the meaningful root. ${ }^{4}$ We then obtain $\varepsilon_{s}=D\left(\bar{k}_{s}, \bar{\omega}_{s}\right)$, which reads, for a lossless structure,

$$
\varepsilon_{s}=\frac{27(1+\delta)^{6} \Lambda^{4}}{P^{3}}
$$

where

$$
\begin{aligned}
& \bar{k}_{s}=1+\frac{1}{2 P}[1-2 \Lambda(1+\delta)], \\
& \bar{\omega}_{s}=(1+\delta)\left[1+\frac{1}{2 P}[1-8 \Lambda(1+\delta)]\right],
\end{aligned}
$$
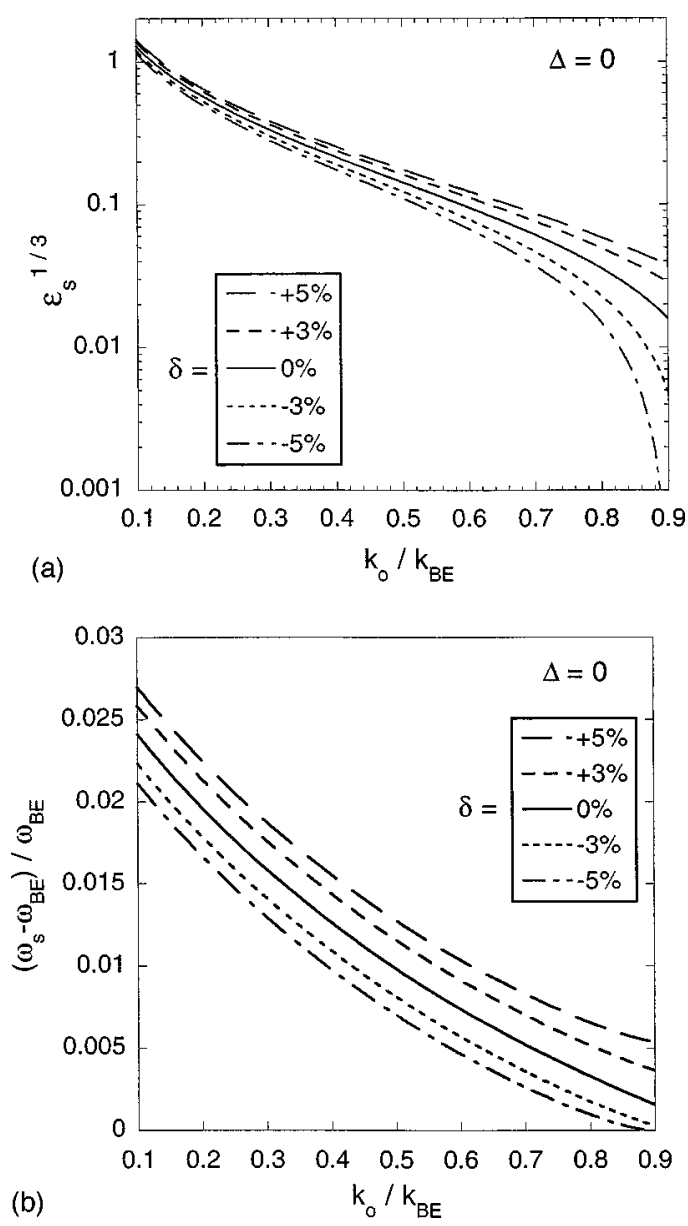

FIG. 2. (a) The threshold coupling constant, $\varepsilon_{s}^{1 / 3}$ as a function of $k_{0} / k_{\mathrm{BE}}$ for a lossless structure $(\Delta=0)$ at various $\delta$. (From top to bottom, $\delta=+5 \%$, $+3 \%, 0 \%,-3 \%,-5 \%$.) (b) The frequency of oscillation at the onset of absolute instability, expressed in terms of the fractional departure from the bandedge frequency $\omega_{\mathrm{BE}}$.

$$
\Lambda=\sqrt{1+\frac{1+2 \delta(1+2 P)}{8(1+\delta)^{2}}}-1
$$

It is obvious from Eq. (3) that $\varepsilon_{s}$ decreases with increasing $P$. This occurs when $k_{0}$ approaches the bandedge wave number $k_{\mathrm{BE}}$ [cf. Eq. (2)]. Note from Eqs. (6) and (3) that when $\delta=-1 / 2(1+2 P), \Lambda=0$ and $\varepsilon_{s}$ becomes zero. This case of zero threshold current may be shown to correspond to the beam mode intersecting the structure mode at the bandedge $\left(v=v_{\mathrm{BE}}\right.$, see Fig. 1). At synchronism ( $\left.\delta=0\right)$, Eqs. (3) $-(5)$ yield $\bar{k}_{s}=1+0.4393 / P, \quad \bar{\omega}_{s}=1+0.2574 / P$, and $\varepsilon_{s}=3.66$ $\times 10^{-4} / P^{3}$. In Fig. 2(a), $\varepsilon_{s}^{1 / 3}$ for $\Delta=0$ is shown as a function of $k_{0} / k_{\mathrm{BE}}$ at various $\delta$, the corresponding frequency of oscillation at the onset is shown in Fig. 2(b). From Fig. 2(a), we see that the threshold increases (decreases) with positive (negative) $\delta$, and becomes very small as $k_{0}$ approaching $k_{\mathrm{BE}}$. The frequency of oscillation, $\omega_{s}$, is in the vicinity of the bandedge frequency $\omega_{\mathrm{BE}}$ [Fig. 2(b)].

The analysis for a lossy structure $(\Delta>0)$ is more involved. Here, $\bar{\omega}_{s}$ remains real and $\bar{k}_{s}$ becomes complex at the onset of absolute instability. In this case, we first find a relation $\bar{k}=\bar{k}(\bar{\omega})$ from the condition $\partial D / \partial \bar{k}=0$. For real values 


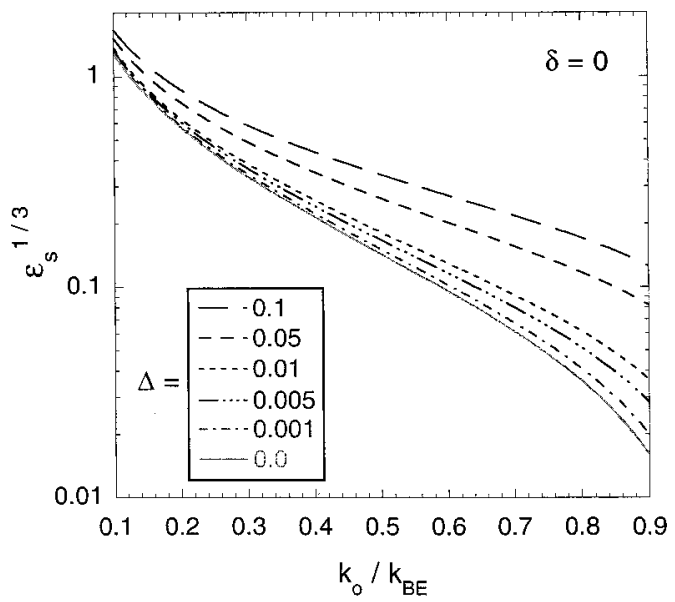

FIG. 3. The threshold coupling constant, $\varepsilon_{s}^{1 / 3}$ as a function of $k_{0} / k_{\mathrm{BE}}$ for a lossy structure with $\delta=0$ at various $\Delta$. (From top to bottom, $\Delta=0.1,0.05$, $0.01,0.005,0.001,0$.

of $\bar{\omega}$, this function $\bar{k}(\bar{\omega})$ is in general complex; so is $\varepsilon$ $=D(\bar{k}(\bar{\omega}), \bar{\omega})$. The critical value of $\varepsilon$ is obtained for that real value of $\bar{\omega}$ at which $\operatorname{Re}[D(\bar{k}(\bar{\omega}), \bar{\omega})]>0$ and $\operatorname{Im}[D(\bar{k}(\bar{\omega}), \bar{\omega})]=0$. We have checked that in the limit $\Delta=0$ (lossless case), this numerical procedure yields the same results as those derived analytically for the lossless case given above. In Fig. $3, \varepsilon_{s}^{1 / 3}$ for $\delta=0$ is shown as a function of $k_{0} / k_{\mathrm{BE}}$ at various $\Delta$. Here, we see that the presence of loss $(\Delta>0)$ raises the threshold current for absolute instability. The stabilization of the bandedge oscillation is more effective at larger $k_{0} / k_{\mathrm{BE}}$. In Fig. 4 , the dependence of $\varepsilon_{s}^{1 / 3}, \bar{\omega}_{s}$ and $\bar{k}_{s}$ (real and imaginary part) on $k_{0} / k_{\mathrm{BE}}$ is shown for the case $\Delta=0.001$ and $\delta=0$.

The above analysis may offer some guidance on improving the stability of TWT, in terms of the required distributed loss and, more importantly, of the separation between the

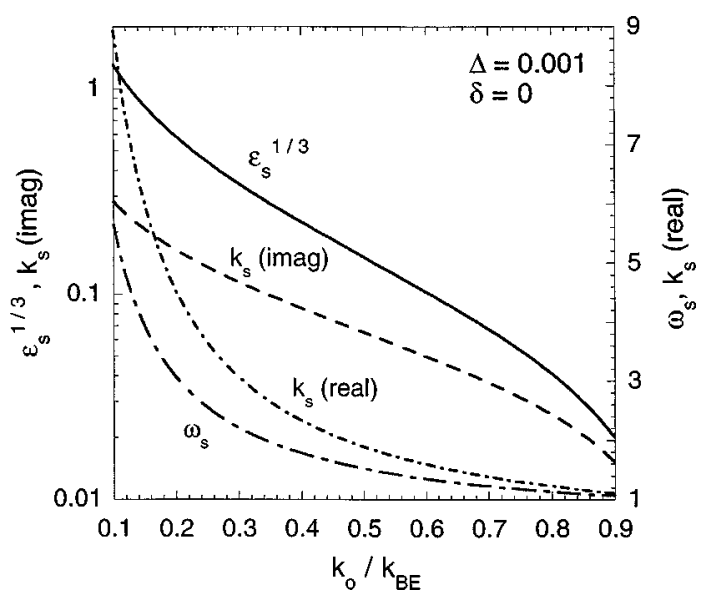

FIG. 4. $\varepsilon_{s}^{1 / 3}, \bar{\omega}_{s}$ and $\bar{k}_{s}$ (real part and imaginary part) as a function of $k_{0} / k_{\mathrm{BE}}$ for a lossy structure with $\Delta=0.001$ and $\delta=0$. $\pi$-mode frequency and the amplifying band. The analytic solution provides a scaling on the gain parameter. Because of the sensitivity to the parameter $P$, which is a measure of the separation between the synchronous mode and the $\pi$-mode wave numbers [cf. Eq. (2)], $P$ needs to be assigned empirically for the tube under study.

\section{CONCLUDING REMARKS}

Finally, we note some differences between absolute instability in fast wave TWT and in slow wave TWT. In a fast wave TWT, such as the gyrotron ${ }^{4,6}$ or gyropeniotron, ${ }^{9}$ absolute instability always occurs near the waveguide cutoff (with zero group velocity), at which the axial wave number is close to zero. Thus, absolute instability in a fast wave tube is quite sensitive to the axial length of the tube, and may not be as readily detected. ${ }^{4}$ On the other hand, absolute instability in a slow wave TWT occurs near the $\pi$-mode (also with zero group velocity ${ }^{7}$ ), whose axial wavelength is much shorter than the axial length of the tube. Thus, absolute instability in a conventional TWT is much less sensitive to the length of the tube, and is much more readily observed.

\section{ACKNOWLEDGMENTS}

We wish to thank David Chernin, Carter Armstrong, Mark Basten, Baruch Levush, and Mark Baird for many useful discussions.

This work was supported by the Multidisciplinary University Research Initiative (MURI), managed by the Air Force Office of Scientific Research and subcontracted through Texas Tech University, by the Northrop Grumman Industrial Associates Program, and by the Naval Research Laboratory.

${ }^{1}$ D. R. Whaley, C. M. Armstrong, B. Gannon, G. Groshart, E. Hurt, J. Hutchins, M. Roscoe, T. M. Antonsen, Jr., and B. Levush, IEEE Trans. Plasma Sci. 26, 912 (1998).

${ }^{2}$ A. S. Gilmour, Traveling Wave Tubes (Artech, Norwood, MA, 1994); B. Epsztein, in IEEE International Electron Device Meeting, Technical Digest, p. 486 (1984), IEEE Catalogue No. CH2099-0/84 (Institute of Electrical and Electronics Engineers, Piscataway, NJ, 1984).

${ }^{3}$ R. J. Briggs, Electron Stream Interaction with Plasma (MIT Press, Cambridge, MA, 1964), Chap. 2; A. Bers, in Plasma Physics-Les Houches 1972, edited by C. DeWitt and J. Peyraud (Gordon and Breach, New York, 1975), p. 113.

${ }^{4}$ Y. Y. Lau, K. R. Chu, L. R. Barnett, and V. L. Granastein, Int. J. Infrared Millim. Waves 2, 373 (1981).

${ }^{5}$ S. M. Miller, T. M. Antonsen, B. Levush, A. Bromborsky, D. K. Abe, and Y. Carmel, Phys. Plasmas 1, 730 (1994).

${ }^{6}$ L. R. Barnett, L. H. Chang, H. Y. Chen, K. R. Chu, W. K. Lau, and C. C. Tu, Phys. Rev. Lett. 63, 1062 (1989).

${ }^{7}$ Strictly speaking, for a perfect helix, the circuit mode does not have a zero group velocity at the $\pi$-mode wavenumber $\pi / L$. See, e.g., Fig 2.9 of D. A. Watkins, Topics in Electromagnetic Theory (Wiley, New York, 1958). However, any slight imperfection in the helix, which is unavoidable in practice, will make $k_{\mathrm{BE}}=\pi / L$ a zero group velocity wavenumber. We wish to thank David Chernin for pointing this out to us. Carter Armstrong has also kindly informed us that oscillations at the $\pi$-mode are of serious concern in the design of TWT.

${ }^{8}$ Y. Y. Lau and D. Chernin, Phys. Fluids B 4, 3473 (1992).

${ }^{9}$ A. K. Ganguly, Y. Y. Lau, and S. Ahn, Phys. Fluids B 4, 3800 (1992). 